\title{
Extraction of CKM matrix elements from lattice QCD results using dispersion relations
}

\section{De-Liang Yao*}

School of Physics and Electronics, Hunan University, 410082 Changsha, China

E-mail: yaodeliang@hnu.edu.cn

\begin{abstract}
The form factors in the semileptonic decays of heavy $B / D$ meson decays can be represented by dispersion relations, by which the kinematics in the decay region are related to the ones in the scattering region. By fitting to the lattice QCD data on the form factors, the subtraction constants in their dispersive representations can be determined such that the form factors in the whole kinematical region are obtained. Cabibbo-Kobayashi-Maskawa elements can be extracted with form-factor values at $q^{2}=0$.
\end{abstract}

Wuhan, China

${ }^{*}$ Speaker. 


\section{Introdcution}

Exclusive semileptonic decays play a crucial role in the determination of Cabibbo-KobayashiMaskawa (CKM) matrix element [1], which is of particular importance to test the standard model (SM) since any effects of violation of unitarity of the CKM matrix could be regarded as signals of new physics beyond SM. Nevertheless, there exist tensions between the values of CKM matrix elements extracted from exclusive and inclusive processes, see e.g. Ref [2] and the references therein. Here we intend to review the work done in Refs. [3, 4, 5] regarding the extraction of the CKM matrix elements from lattice QCD results using dispersion relations.

One of the simplest types of semileptonic decays is $H(p) \rightarrow H^{\prime}\left(p^{\prime}\right) \ell\left(p_{\ell}\right) \bar{v}_{\ell}\left(p_{v}\right)$, where $\ell, \bar{v}_{\ell}$, $H$ and $H^{\prime}$ stand for lepton, anti-neutrino, initial and final hadrons, respectively. Under one-boson exchange approximation, the corresponding invariant amplitude reads

$$
\mathscr{M}=\frac{G_{F} V_{q^{\prime} q}}{\sqrt{2}}\left\{\bar{u}\left(p_{\ell}\right) \gamma^{\mu}\left(1-\gamma_{5}\right) v\left(p_{v}\right)\right\}\left\{\left\langle H^{\prime}\left(p^{\prime}\right)\left|\bar{q}^{\prime} \gamma_{\mu}\left(1-\gamma_{5}\right) q\right| H(p)\right\rangle\right\},
$$

where $V_{q^{\prime} q}$ represents the element of CKM matrix and $G_{F}=1.166 \times 10^{-5} \mathrm{GeV}^{-2}$ is the Fermi constant. In above, the term in the first curly braces is called the leptonic part which is well-known in the sense that it can be straightforwardly calculated in Standard Model. In contrast, the hadronic part in the second curly braces is usually difficult to be directly derived in Quantum Chromodynamics (QCD) due its non-perturbative property at low energies. In the low-energy region, one needs to adopt approaches, such as lattice QCD, effective field theories or phenomenological models, to extract information on the hadronic part.

In the case that both $H$ and $H^{\prime}$ are mesons with quantum numbers $J^{P}=0^{-}$, the hadronic part in Eq. (1.1) can be decomposed as

$$
\left\langle H^{\prime}\left(p^{\prime}\right)\left|\bar{q}^{\prime} \gamma^{\mu}\left(1-\gamma^{5}\right) q\right| H(p)\right\rangle=f_{+}\left(q^{2}\right)\left[P^{\mu}-\frac{m_{H}^{2}-m_{H^{\prime}}^{2}}{q^{2}} q^{\mu}\right]+f_{0}\left(q^{2}\right) \frac{m_{H}^{2}-m_{H^{\prime}}^{2}}{q^{2}} q^{\mu},
$$

with $q^{\mu} \equiv p^{\prime \mu}-p^{\mu}$ and $P^{\mu} \equiv\left(p+p^{\prime}\right)^{\mu} . m_{H}$ and $m_{H^{\prime}}$ are masses of the initial and final hadrons, respectively. The piece proportional to the $\gamma^{5}$ does not contribute according to parity conservation. Here $f_{0}\left(q^{2}\right)$ and $f_{+}\left(q^{2}\right)$ are called scalar and vector form factors, respectively, satisfying the kinematical constraint $f_{+}(0)=f_{0}(0)$ at $q^{2}=0$. Note that the kinematic range of the four-momentum transfer squared $q^{2}$ is $\left[q_{\min }^{2} \equiv m_{\ell}^{2}, q_{\max }^{2} \equiv\left(m_{H}-m_{H^{\prime}}\right)^{2}\right]$.

In the rest frame of the initial meson, the differential decay rate can be expressed as

$$
\begin{gathered}
\frac{\mathrm{d} \Gamma}{\mathrm{d} q^{2}}=\frac{G_{F}^{2}}{96 \pi^{3}} \eta_{W}^{2}\left|V_{q^{\prime} q}\right|^{2} \frac{q^{2}}{m_{H}^{2}}\left[1-\frac{m_{\ell}^{2}}{q^{2}}\right]\left|\vec{p}^{*}\right|\left[\left(1+\frac{m_{\ell}^{2}}{2 q^{2}}\right)\left|H_{0}\right|^{2}+\frac{3 m_{\ell}^{2}}{2 q^{2}}\left|H_{t}\right|^{2}\right], \\
H_{0}=\frac{2 m_{H}|\vec{p}|^{*}}{\sqrt{q^{2}}} f_{+}\left(q^{2}\right), \quad H_{t}=\frac{m_{H}^{2}-m_{H^{\prime}}^{2}}{\sqrt{q^{2}}} f_{0}\left(q^{2}\right),
\end{gathered}
$$

where $\eta_{W}$ accounts for electroweak corrections and $m_{\ell}$ denotes the mass of the lepton. The differential decay rate can be measured by experiments. Once the form factors are known, the CKM matrix element $V_{q^{\prime} q}$ can be readily determined.

In the energy region close to $q_{\max }^{2}$, the form factors are extensively studied by various lattice QCD groups, see Ref. [6] for a review. While in the region close to $q_{\min }^{2}$, there are works investigating the form factors by using light cone sum rules (LCSR) and perturbative QCD (pQCD). In 
our works $[3,4,5]$, dispersion techniques are utilized in order to describe the form factors in the whole kinematic region. Furthermore, dispersion relations build an elegant bridge to connect decay form factors with scattering amplitudes, which is helpful to communicate information each other.

\section{Heavy-to-light semileptonic decays}

Heavy-to-light semileptonic decays are those processes with $H \in\{D, \bar{B}\}$ and $H^{\prime} \in\{\pi, K, \bar{K}, \eta\}$ in Eq. (1.2). In Ref. [4], the scalar form factors in the heavy-to-light semileptonic decays $D \rightarrow \bar{K} \bar{\ell} v_{\ell}$, $D \rightarrow \pi \bar{\ell} v_{\ell}, \bar{B}_{s} \rightarrow K \ell \bar{v}_{\ell}$, and $\bar{B} \rightarrow \pi \ell \bar{v}_{\ell}$ are simultaneously explored by using Muskhelishvili-omnès (MO) dispersion relations.

To be specific, the scalar form factors, collected in a multiplet $\overrightarrow{\mathscr{F}}$ according to strangeness $S$ and isospin $I$, are expressed by

$$
\overrightarrow{\mathscr{F}}(s)=\Omega(s) \cdot \overrightarrow{\mathscr{P}}(s), \quad s \equiv q^{2}
$$

where $\Omega(s)$ is MO matrix and $\overrightarrow{\mathscr{P}}(s)$ is a vector of polynomial components with real coeffecients. The MO matrix satisfies an un-subtracted dispersion relation $[7,8]$,

$$
\Omega(s)=\frac{1}{\pi} \int_{s_{\mathrm{th}}}^{\infty} \frac{\mathbf{T}^{*}(s) \Sigma(s) \Omega(s)}{s^{\prime}-s-i \varepsilon} \mathrm{d} s^{\prime},
$$

with $s_{\text {th }}$ being the lowest threshold. Here, $\mathbf{T}$ is the coupled channel $S$-wave $H H^{\prime}$ scattering amplitudes and $\Sigma(s)=\operatorname{diag}\left\{\sigma_{H_{1} H_{1}^{\prime}}, \cdots, \sigma_{H_{n} H_{n}^{\prime}}\right\}$ with $\sigma_{H_{i} H_{i}^{\prime}}=\sqrt{\left[s-\left(m_{H_{i}}+m_{H_{i}^{\prime}}\right)^{2}\right]\left[s-\left(m_{H_{i}}-m_{H_{i}^{\prime}}\right)^{2}\right]} / s$ and $n$ the number of channels.

The integral equation in Eq. (2.2) can be solved by following the numerical method given in Ref. [9]. As input, the $T$-matrix is needed. To that end, the scattering amplitudes based on unitarized chiral effective theory calculated in Refs. [10, 11] are used. Actually, the most recent unitarized amplitudes based on one-loop potentials are computed in Refs. [12, 13] but are not employed for the reason that the involved LECs at one-loop level can not be very well determined due to the lack of precise data, as it is pointed out in Ref. [14]. The unitarized $T$-matrices are only valid in the energy region not far from thresholds, namely, only valid up to a certain value of $s$, denoted by $s_{m}$. In the region between $s=s_{m}$ and $s=\infty$, we assume $T$-matrix elements satisfy certain asymptotic conditions which guarantee the MO equations have unique solutions, see Ref. [4] for details and especially for numerical solutions for the MO matrices in each channel.

According to Eq. (2.1), once the Omnès matrix is obtained, the form factor $\overrightarrow{\mathscr{F}}(s)$ is determined up to a polynomial $\overrightarrow{\mathscr{P}}(s)$ which contains some unknown coefficients. To reduce the number of unknown parameters, the dispersive representations of the scalar form factors can be matched to next-to-leading order (NLO) chiral representations of the form factors in a kinematic region where the latter are still valid. The NLO chiral representations can be derived in heavy meson chiral perturbation theory by imposing the chiral Lagrangians given in Refs. [15, 16, 17].

Now, one is in the position to perform fits to Lattice QCD data on the scalar form factors. A combined bottom-charm fit is performed to Lattice QCD and LCSR data. For the bottom sector, the data are taken form UKQCD [18], HPQCD [19, 20] and Fermilab Lattice \& MILC (labeled as FLMILC for short) [21]) and LCSR [22, 23] results for the scalar form factors in $\bar{B}^{0} \rightarrow \pi^{+}$and $\bar{B}_{s}^{0} \rightarrow$ $\mathrm{K}^{+}$semileptonic decays. In the charm sector, the recent data along with the covariance matrices 
provided by the ETM Collaboration [24] are used. Note that Lorentz symmetry breaking due to hypercubic effects is clearly seen in the ETM data and has been included in the decomposition of the current matrix elements [24]. However, those discretization errors are not considered in the HPQCD studies [25, 26], and hence the HPQCD results are excluded in the combined fit. Fit results are shown in Fig. 1.
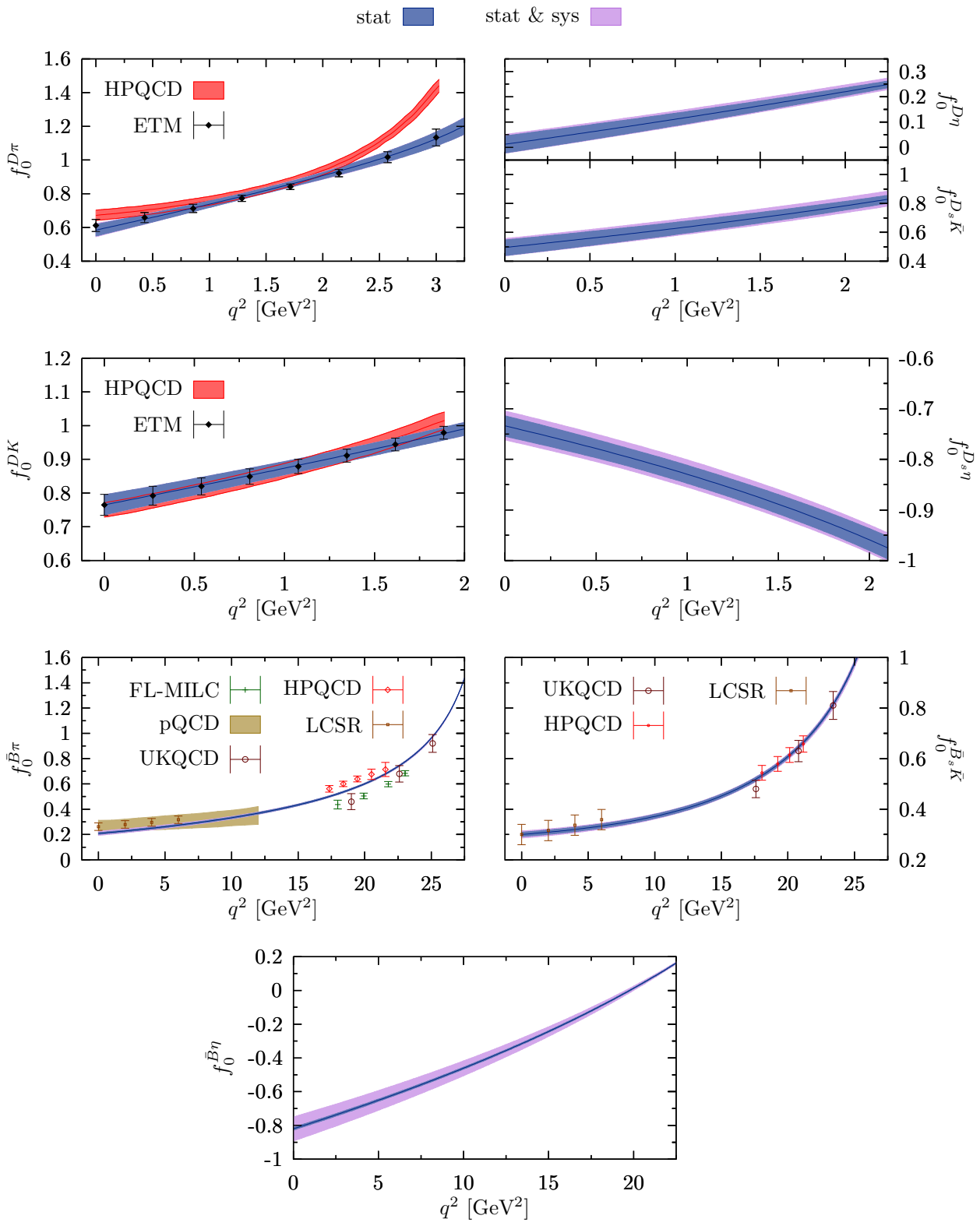

Figure 1: Predictions of scalar form factors based on the combined fit. The predictions for the $D \rightarrow \eta$, $D_{s} \rightarrow K$ and $D_{s} \rightarrow \eta$ scalar form factors are obtained by chiral symmetry. For comparison, we show results from the HPQCD [25, 26] Collaboration. One can find visible differences between ETM and HPQCD sets of $D \rightarrow \pi$ and $D \rightarrow K$ form factors in the vicinity of $q_{\max }^{2}$, particularly for the $D \rightarrow \pi$ case. 
From the combined fit displayed in Fig. 1, one obtains

$$
\begin{aligned}
f_{+}^{D \rightarrow \pi}(0) & =0.585(35)_{\text {stat }}(19)_{\text {sys }_{1}}(32)_{\mathrm{sys}_{2}} \\
f_{+}^{D \rightarrow \bar{K}}(0) & =0.765(30)_{\text {stat }}(4)_{\text {sys }_{1}}(14)_{\text {sys }_{2}} \\
f_{+}^{\bar{B} \rightarrow \pi}(0) & =0.208(7)_{\text {stat }}(15)_{\text {sys }_{1}}(30)_{\text {sys }_{2}}
\end{aligned}
$$

where the first and second sets of errors account for statistical and chiral systematical uncertainties propagated from the errors of the LECs involved in the MO matrix, respectively. The third errors takes into account the variations that are produced when in the best fit one considers alternatively only HPQCD or UKQCD and FL-MILC $\bar{B} \rightarrow \pi$ form factors. In combination with the following experimental values

$$
\begin{aligned}
& f_{+}^{D \rightarrow \pi}(0)\left|V_{c d}\right|=0.1426(19), \\
& f_{+}^{D \rightarrow \bar{K}}(0)\left|V_{c s}\right|=0.7226(34),
\end{aligned}
$$

taken from the report by the Heavy Flavor Averaging Group (HFLAV) [27], and

$$
f_{+}^{\bar{B} \rightarrow \pi}(0)\left|V_{u b}\right|=(8.9 \pm 0.3) \times 10^{-4},
$$

which is an average value of the Belle and BaBar results $(9.2 \pm 0.3) \times 10^{-4}[28]$ and $(8.7 \pm 0.3) \times$ $10^{-4}$ [29], we eventually obtain the following values for the corresponding CKM matrix elements.

$$
\begin{aligned}
\left|V_{c d}\right| & =0.244(22), \\
\left|V_{c s}\right| & =0.945(41), \\
10^{3}\left|V_{u b}\right| & =4.3(7) .
\end{aligned}
$$

As byproducts, we also predict the absolute values of the vector form factors at $q^{2}=0$ for the channels related by chiral symmetry

$$
\begin{aligned}
\left|f_{+}^{\bar{B}_{s} \rightarrow K}(0)\right| & =0.301(9)_{\mathrm{stat}}(11)_{\mathrm{sys}_{1}}(26)_{\mathrm{sys}_{2}} \\
\left|f_{+}^{\bar{B} \rightarrow \eta}(0)\right| & =0.82(1)_{\text {stat }}(7)_{\mathrm{sys}_{1}}(3)_{\mathrm{sys}_{2}} \\
\left|f_{+}^{D_{s} \rightarrow \eta}(0)\right| & =0.734(21)_{\mathrm{stat}}(21)_{\mathrm{sys}_{1}}(3)_{\mathrm{sys}_{2}} \\
\left|f_{+}^{D_{s} \rightarrow K}(0)\right| & =0.50(6)_{\text {stat }}(3)_{\mathrm{sys}_{1}}(5)_{\mathrm{sys}_{2}} \\
\left|f_{+}^{D \rightarrow \eta}(0)\right| & =0.01(3)_{\text {stat }}(2)_{\text {sys }_{1}}(4)_{\mathrm{sys}_{2}}
\end{aligned}
$$

\section{A new parametrization for $\bar{B} \rightarrow D \ell \bar{v}_{\ell}$}

We discussed heavy-to-light semileptonic decays in the above section, now we move to a heavy-to-heavy semileptonic decay with $H=\bar{B}$ and $H^{\prime}=D$. A new parametrization is proposed for $\bar{B} \rightarrow D \ell \bar{v}_{\ell}$ in Ref. [5]. Let us first review the formalism. Thanks to analyticity, unitarity and crossing symmetry, the form factors in $\bar{B} \rightarrow D \ell \bar{v}_{\ell}$ can be written in the Omnès form

$$
f_{i}\left(q^{2}\right)=f_{i}\left(s_{0}\right) \exp \left[\frac{q^{2}-s_{0}}{\pi} \int_{s_{\mathrm{th}}}^{\infty} \frac{\mathrm{d} s}{s-s_{0}} \frac{\alpha^{i}(s)}{s-q^{2}}\right], \quad i=+, 0
$$


Provided that $s \geq s_{\mathrm{th}} \gg q_{\max }^{2} \geq q^{2}$, the above equation can be written as a new form,

$$
f_{i}\left(q^{2}\right)=f_{i}\left(s_{0}\right) \prod_{n=0}^{\infty} \exp \left[\frac{q^{2}-s_{0}}{s_{\mathrm{th}}} \mathscr{A}_{n}^{i} \frac{q^{2 n}}{s_{\mathrm{th}}^{n}}\right], \quad \mathscr{A}_{n}^{i} \equiv \frac{1}{\pi} \int_{s_{\mathrm{th}}}^{+\infty} \frac{\mathrm{d} s}{s-s_{0}} \frac{\alpha^{i}(s)}{\left(s / s_{\mathrm{th}}\right)^{n+1}} .
$$

The coefficients $\mathscr{A}_{n}^{i}$ are called phase moments and are related to the phases of the amplitudes in the physical $\bar{B} \bar{D}$ scattering region. Imposing the new parametrization in Eq. (3.2), lattice QCD date on the form factors by FL-MILC collaborations [30] and HPQCD [31] together with experimental data on differential decay rate measured by Belle [32] are fitted. The fit results are displayed in Fig. 2. Based on the best fit, we get

$$
\left|V_{c b}\right|=(41.01 \pm 0.75) \times 10^{-3} .
$$
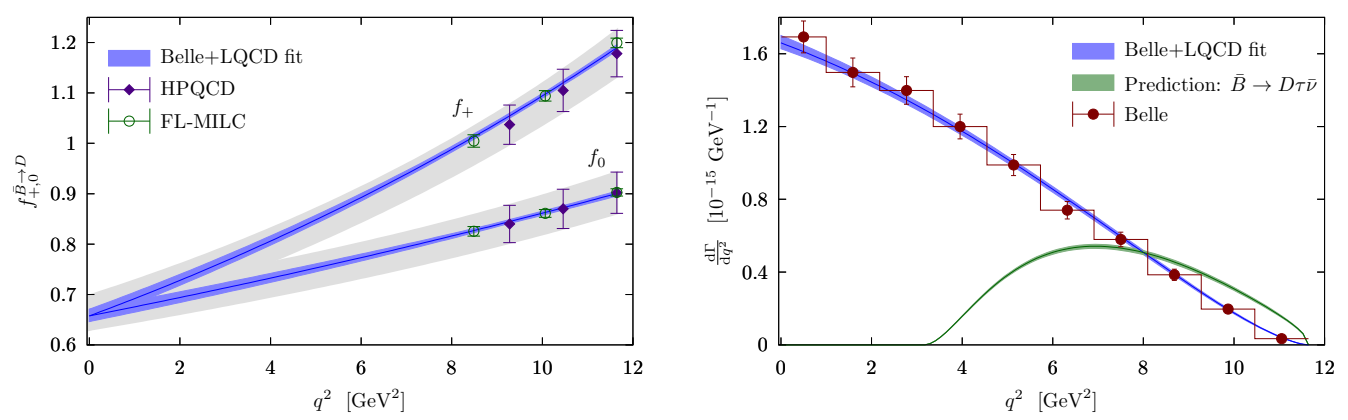

Figure 2: Left panel: vector and scalar form factors. Right panel: differential decay rates. The gray bands stand for the HPQCD results from the BCL continuous parametrization provided in [31].

\section{Summary and outlook}

The extraction of CKM matrix elements from lattice QCD results using dispersion relations, which are presented in detail in Refs. [3, 4, 5], has been reviewed. Lattice QCD and experimental data are fitted to pin down all the involved unknown parameters such that the CKM matrix elements can be predicted. A new parametrization for $\bar{B} \rightarrow D \ell \bar{v}_{\ell}$ is proposed, which can be also used to investigate $\bar{B} \rightarrow D^{*} \ell \bar{v}_{\ell}$ and $\bar{\Lambda}_{b} \rightarrow \Lambda_{c} \ell \bar{v}_{\ell}$ decays in future.

\section{Acknowledgments}

I would like to thank M. Albaladejo, P. Fernandez-Soler, F.-K. Guo and J. Nieves for sharing their insights into this project. This work of DLY has been supported by National Science Foundation of China under Grant No. 11905258.

\section{References}

[1] J. D. Richman and P. R. Burchat, Rev. Mod. Phys. 67, 893 (1995).

[2] Y. S. Amhis et al. [HFLAV Collaboration], arXiv:1909.12524 [hep-ex]. 
[3] D. L. Yao, M. Albaladejo, P. Fernández-Soler, F. K. Guo and J. Nieves, PoS Hadron 2017, 089 (2018).

[4] D. L. Yao, P. Fernandez-Soler, M. Albaladejo, F. K. Guo and J. Nieves, Eur. Phys. J. C 78, no. 4, 310 (2018).

[5] D. L. Yao, P. Fernandez-Soler, F. K. Guo and J. Nieves, arXiv:1906.00727 [hep-ph].

[6] S. Aoki et al., Eur. Phys. J. C 77, no. 2, 112 (2017).

[7] R. Omnes, Nuovo Cim. 8, 316 (1958).

[8] N. Muskhelishvili, Singular integral equations. Boundary problems of function theory and their application to mathematical physics, Groningen/Holland: P. Noordhoff, (1953).

[9] B. Moussallam, Eur. Phys. J. C 14, 111 (2000).

[10] F. K. Guo, C. Hanhart, S. Krewald and U. G. Meißner, Phys. Lett. B 666, 251 (2008).

[11] L. Liu, K. Orginos, F. K. Guo, C. Hanhart and U. G. Meißner, Phys. Rev. D 87, no. 1, 014508 (2013).

[12] D. L. Yao, M. L. Du, F. K. Guo and U. G. Meißner, JHEP 1511, 058 (2015).

[13] M. L. Du, F. K. Guo, U. G. Meißner and D. L. Yao, Eur. Phys. J. C 77, no. 11, 728 (2017).

[14] M. L. Du, F. K. Guo, U. G. Meißner and D. L. Yao, Phys. Rev. D 94, no. 9, 094037 (2016).

[15] M. B. Wise, Phys. Rev. D 45, no. 7, R2188 (1992).

[16] G. Burdman and J. F. Donoghue, Phys. Lett. B 280, 287 (1992).

[17] T. M. Yan, H. Y. Cheng, C. Y. Cheung, G. L. Lin, Y. C. Lin and H. L. Yu, Phys. Rev. D 46, 1148 (1992) Erratum: [Phys. Rev. D 55, 5851 (1997)].

[18] J. M. Flynn, T. Izubuchi, T. Kawanai, C. Lehner, A. Soni, R. S. Van de Water and O. Witzel, Phys. Rev. D 91, no. 7, 074510 (2015).

[19] E. Dalgic, A. Gray, M. Wingate, C. T. H. Davies, G. P. Lepage and J. Shigemitsu, Phys. Rev. D 73, 074502 (2006) Erratum: [Phys. Rev. D 75, 119906 (2007)].

[20] C. M. Bouchard et al., Phys. Rev. D 90, 054506 (2014).

[21] J. A. Bailey et al. [Fermilab Lattice and MILC Collaborations], Phys. Rev. D 92, no. 1, 014024 (2015).

[22] G. Duplancic, A. Khodjamirian, T. Mannel, B. Melic and N. Offen, JHEP 0804, 014 (2008).

[23] G. Duplancic and B. Melic, Phys. Rev. D 78, 054015 (2008).

[24] V. Lubicz et al. [ETM Collaboration], Phys. Rev. D 96, no. 5, 054514 (2017) Erratum: [Phys. Rev. D 99, no. 9, 099902 (2019)].

[25] H. Na, C. T. H. Davies, E. Follana, G. P. Lepage and J. Shigemitsu, Phys. Rev. D 82, 114506 (2010).

[26] H. Na, C. T. H. Davies, E. Follana, J. Koponen, G. P. Lepage and J. Shigemitsu, Phys. Rev. D 84, 114505 (2011).

[27] Y. Amhis et al. [HFLAV Collaboration], Eur. Phys. J. C 77, no. 12, 895 (2017).

[28] H. Ha et al. [Belle Collaboration], Phys. Rev. D 83, 071101 (2011).

[29] J. P. Lees et al. [BaBar Collaboration], Phys. Rev. D 86, 092004 (2012).

[30] J. A. Bailey et al. [MILC Collaboration], Phys. Rev. D 92, no. 3, 034506 (2015).

[31] H. Na et al. [HPQCD Collaboration], Phys. Rev. D 92, no. 5, 054510 (2015) Erratum: [Phys. Rev. D 93, no. 11, 119906 (2016)].

[32] R. Glattauer et al. [Belle Collaboration], Phys. Rev. D 93, no. 3, 032006 (2016). 